\title{
R13 Moment Equations Applied to Supersonic Flow with Solid Wall Interaction
}

\author{
Maksim Timokhin $^{1,2, a)}$, Henning Struchtrup ${ }^{3, b)}$, Alexey Kokhanchik ${ }^{4}$ and Yevgeniy \\ Bondar $^{4, c)}$ \\ ${ }^{1}$ Lomonosov Moscow State University, 119991, Moscow, Russia \\ ${ }^{2}$ Moscow Aviation Institute, 125993, Moscow, Russia \\ ${ }^{3}$ Department of Mechanical Engineering University of Victoria, Victoria, BC, Canada \\ ${ }^{4}$ Khristianovich Institute of Theoretical and Applied Mechanics, 630090, Novosibirsk, Russia \\ ${ }^{a)}$ Corresponding author: timokhin@physics.msu.ru \\ b) struchtr@uvic.ca \\ c) bond@itam.nsc.ru
}

\begin{abstract}
R13 moment set of equations is the regularization of original Grad 13-moment system. Several variants of R13 equations were presented after the first publication. The difference between them lies in the relations for higher order moments. It has been shown that all variants are well applicable for numerical modelling of the moderately rarefied slow gas flows and allow the modelling of main nonequilibrium effects in micro-flow problems. The difficulty is that the solid wall boundary conditions for R13 equations were obtained from one of the variants which is not applicable to near hypersonic flows. This study is devoted to the demonstration of the numerical results of R13 linear and nonlinear variants for supersonic flow over the flat plate. The R13 numerical solutions are compared with DSMC results computed by the SMILE++ software system.
\end{abstract}

\section{INTRODUCTION}

In 1949 Grad proposed original 13 moment system of equations which was derived from the kinetic Boltzmann equation due to Hermite polynomial expansion of velocity distribution function around Maxwellian [1]. This system is written with respect to macroparameters (moments of the distribution function) and describes the gas not only when it can be considered as continuum, but also in the transitional flow regime, i.e., it takes rarefaction effects into account. In the general case, the system of moment equations is infinite. Some additional closure relations are introduced to obtain a finite system. Thus, higher-order moments that are not included into the system are expressed in terms of moments retained in the system. It turned out later, however, that Grads method has some drawbacks. It was shown [2, 3] that the series in the Hermite polynomials representing the distribution function in the shock wave does not converge in the case of a finite radius of molecular interaction. As a result, there appear unphysical jumps of gas-dynamic parameters in supersonic flows at Mach numbers greater than 1.65 [4, 5]. In this work, we consider 13-moment Grad system with its regularization proposed by Struchtrup and Torrilhon [6] (or R13 equations). The regularization of original system implies that high-order moments are presented in the form of expressions containing 13 moments (density, three components of velocity, six components of the symmetric stress tensor, and three components of the heat flux) and their gradients. It was shown the solution of the resultant system of equations does not contain unphysical jumps of gasdynamic parameters inside the shock wave front at Mach numbers greater than 1.65 [5]. Later on, the regularization procedure proposed by Struchtrup and Torrilhon was also applied for the 20-moment and 26-moment systems of Grad equations [7].

During last 15 years several variants of R13 equations were presented after the first publication [6]. The difference between them lies in the relations for higher order moments $[8,9]$. It has been shown that all variants are well applicable for numerical modelling of the moderately rarefied slow gas flows and allow the modelling of main nonequilibrium effects in low-velocity micro-flow problems $[10,11,12,13,14,15,16,17]$ and some moderately 
rarefacted supersonic flows $[5,18,19,15,20,21,22]$. The difficulty is that the solid wall boundary conditions for R13 equations were obtained from one of the variants [10] which is not applicable to near hypersonic flows [9]. This study is devoted to the demonstration of the numerical results of R13 variants (linear and nonlinear) for supersonic flow over the flat plate. It is a classical fluid dynamic problem. Simple geometry of the problem allows to minimize the geometrical factor influence to the investigated flow. Moreover, the comparison of different rarefied gas models for the flow about a flat plate at zero angle of attack is especially interesting due to significant degree of thermal non-equilibrium which is observed in the vicinity of the leading edge at any Knudsen number. In fact, local Knudsen number in the vicinity of the leading edge tends to infinity (because of the leading edge is mathematically sharp) and it is challenging for the gas models to predict the flow in this zone with sufficient accuracy. The applicability of this continuum approach is tested by the comparison with the kinetic solution obtained by DSMC with SMILE++ software [23, 24].

\section{PROBLEM FORMULATION}

The formulation of the problem is presented in Fig. 1. The length of the plate is 1 (from 0.5 to 1.5 coordinates in the bottom). The plate surface is modelled with full accommodation. The rest of bottom part has specular reflection (symmetry plane). The computations were performed in a monatomic gas with hard-sphere molecular interaction $\left(\omega=0.5\right.$ in the power viscosity law) for two cases of the free-stream Mach number $\left(M a_{\infty}=2.0\right.$ and $\left.M a_{\infty}=4.0\right)$. Knudsen number based on the plate length is equal to $\mathrm{Kn}=0.05$ in both cases. The free-stream average free path $\lambda_{\infty}$ is defined by [25]

$$
\lambda_{\infty}=\frac{4 \alpha(5-2 \omega)(7-2 \omega)}{5(\alpha+1)(\alpha+2)} \frac{\mu}{\rho}(2 \pi \theta)^{-\frac{1}{2}},
$$

where $\omega=0.5$ and $\alpha=1.0$.

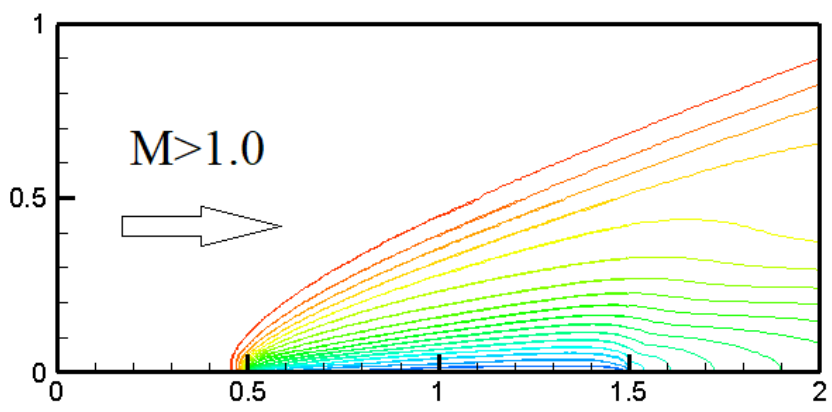

FIGURE 1. The computational domain and flow structure.

\section{R13 EQUATIONS}

\section{Bulk Equations}

The regularization of the Grads original 13-moment system [1,3] was derived in 2003 [6] by a Chapman-Enskog expansion [26] of higher moment equations only, based on the assumption of faster relaxation times for higher moments. Later the derivation of the R13 equations were developed explicitly without this assumption (Order of Magnitude Method) [18]. Nevertheless, the resulting equations are meaningful, and, since they contain some higher order terms. The tensor form of the regularized 13-moment system (R13) can be written as

$$
\begin{gathered}
\frac{\partial \rho}{\partial t}+\frac{\partial \rho v_{k}}{\partial x_{k}}=0 \\
\rho \frac{\partial v_{i}}{\partial t}+\rho v_{k} \frac{\partial v_{i}}{\partial x_{k}}+\frac{\partial p}{\partial x_{i}}+\frac{\partial \sigma_{i k}}{\partial x_{k}}=0
\end{gathered}
$$




$$
\begin{gathered}
\frac{3}{2} \rho \frac{\partial \theta}{\partial t}+\frac{3}{2} \rho v_{k} \frac{\partial \theta}{\partial x_{k}}+\frac{\partial q_{k}}{\partial x_{k}}+p \frac{\partial v_{k}}{\partial x_{k}}+\sigma_{i j} \frac{\partial v_{i}}{\partial x_{j}}=0 \\
\frac{\partial \sigma_{i j}}{\partial t}+\frac{\partial \sigma_{i j} v_{k}}{\partial x_{k}}+\frac{4}{5} \frac{\partial q_{\langle i}}{\partial x_{j\rangle}}+2 p \frac{\partial v_{\langle i}}{\partial x_{j\rangle}}+2 \sigma_{k\langle i} \frac{\partial v_{j\rangle}}{\partial x_{k}}+\frac{\partial m_{i j k}}{\partial x_{k}}=-\frac{\sigma_{i j}}{\tau}, \\
\frac{\partial q_{i}}{\partial t}+\frac{\partial q_{i} v_{k}}{\partial x_{k}}+\frac{5}{2} p \frac{\partial \theta}{\partial x_{i}}+\frac{5}{2} \sigma_{i k} \frac{\partial \theta}{\partial x_{k}}+\theta \frac{\partial \sigma_{i k}}{\partial x_{k}}-\sigma_{i k} \theta \frac{\partial \rho}{\partial x_{k}}-\frac{\sigma_{i j}}{\rho} \frac{\partial \sigma_{j k}}{\partial x_{k}} \\
+\frac{7}{5} q_{k} \frac{\partial v_{i}}{\partial x_{k}}+\frac{2}{5} q_{k} \frac{\partial v_{k}}{\partial x_{i}}+\frac{2}{5} q_{i} \frac{\partial v_{k}}{\partial x_{k}}+\frac{1}{2} \frac{\partial R_{i k}}{\partial x_{k}}+\frac{1}{6} \frac{\partial \Delta}{\partial x_{i}}+m_{i j k} \frac{\partial v_{j}}{\partial x_{k}}=-\frac{2}{3} \frac{q_{i}}{\tau},
\end{gathered}
$$

where the mass density $\rho$, velocity $v_{i}$, temperature $\theta$ in energy units $\theta=\frac{k}{m} T$ ( $k$ is the Boltzmann constant and $m$ the particle mass), trace-free viscous stress tensor $\sigma_{i j}$ (with $\sigma_{i i}=0$ ), and heat flux $q_{i}$ form the 13 primitive variables. The pressure is given by the ideal gas state equation $p=\rho \theta$. The angular brackets in the subscripts indicate the trace-free and symmetric part of the tensor [18]. $\tau$ is the relaxation time, with the dynamic viscosity coefficient $\mu$. This system of equations was derived for the Maxwell model of molecular interaction. At the same time, the system is used for other potentials of molecular interaction with the viscosity law obtained on the basis of these potentials [18]. The viscosity is defined by the power law

$$
\mu=\frac{p}{v}=p \tau=\mu_{r e f}\left(\frac{T}{T_{r e f}}\right)^{\omega},
$$

where $0.5 \leq \omega \leq 1.0$. The values $\omega=0.5$ and $\omega=1.0$ correspond to the models of hard spheres and Maxwell molecules, respectively. As it was mentioned above, we consider the case with $\omega=0.5$.

The equations (2) - (4) present the conservation laws for mass, momentum and energy; equations (5) and (6) are the moment equations for stress tensor and heat flux vector, respectively. These 13 equations must be closed by constitutive relations for the higher moments $R_{i j}, \Delta, m_{i j k}$, and they differ based on the method of regularization. For Grads original 13 moment equations [1], $R_{i j}=\Delta=m_{i j k}=0$. Higher-order moments have the following analytical form [18]:

$$
\begin{gathered}
\Delta=-\frac{\sigma_{k l} \sigma_{k l}}{\rho}+6 \frac{\sigma_{k l} \sigma_{k l}^{N S F}}{\rho}+\frac{56}{5} \frac{q_{k} q_{k}^{N S F}}{p}-12 \mu \theta \frac{\partial}{\partial x_{k}}\left(\frac{q_{k}}{p}\right) \\
R_{i j}=-\frac{4}{7} \frac{\sigma_{k\langle i} \sigma_{j\rangle k}}{\rho}+\frac{24}{7} \frac{\sigma_{k\langle i} \sigma_{j\rangle k}^{N S F}}{\rho}+\frac{64}{25} \frac{q_{\langle i} q_{j\rangle}^{N S F}}{p}-\frac{24}{5} \mu \theta \frac{\partial}{\partial x_{\langle i}}\left(\frac{q_{j\rangle}}{p}\right) \\
m_{i j k}=\frac{8}{15} \frac{\sigma_{\langle i j} q_{k\rangle}^{N S F}}{p}+\frac{4}{5} \frac{q_{\langle i} \sigma_{j k\rangle}^{N S F}}{p}-2 \mu \theta \frac{\partial}{\partial x_{\langle i}}\left(\frac{\partial \sigma_{j k\rangle}}{p}\right)
\end{gathered}
$$

where $\sigma_{i j}^{N S F}$ and $q_{i}^{N S F}$ are the values from the Navier-Stokes and Fourier laws correspondingly:

$$
\sigma_{i j}^{N S F}=-2 \mu \frac{\partial v_{\langle i}}{\left.\partial x_{j}\right\rangle}, q_{i}^{N S F}=-\frac{15}{4} \mu \frac{\partial \theta}{\partial x_{i}}
$$

There are several nonlinear variants of R13 equations which are different in higher order moment relations [6, $18,10,14,9,8]$. In the linear case (the terms presenting only gradient transport mechanism, GTM [7]), higher-order moments have the following form:

$$
m_{i j k}=-2 \tau \frac{k}{m} T \frac{\partial \sigma_{\langle i j}}{\partial x_{k\rangle}}, R_{i j}=-\frac{24}{5} \tau \frac{k}{m} T \frac{\partial q_{\langle i}}{\partial x_{j\rangle}}, \Delta=-12 \tau \frac{k}{m} T \frac{\partial q_{l}}{\partial x_{l}} .
$$

The rest of terms in the relations present, so-called, non-gradient transport mechanism (NGMT, [7]). Their influence increases in the case of strong non-equilibrium presence [9]. All the R13 variants and their applicability evaluation to shock-wave structure problem are presented in $[8,9]$. The linear variant and nonlinear variant obtained by Order of Magnitude Method [18] of R13 equations have been used in present study. 


\section{Solid Wall Boundary Conditions}

The first attempt to derive solid wall boundary conditions for R13 equations was done by Gu and Emerson in [27]. Further the similar method was used by Struchtrup and Torrilhon to derive their version of the boundary conditions [10] on the solid wall. The obtaining of macro-parameter boundary conditions is based on Maxwell accommodative model [28] and the Grad's idea that the weight function in the distribution function moments should be even in the normal component of the particle velocity [29]. The resulting kinetic boundary conditions with wall velocity have following form [10]:

$$
\begin{gathered}
v_{n}=0, \\
v_{\tau}=v_{w}-\frac{1}{p_{\chi}}\left(\sqrt{\frac{\pi}{2} \theta} \frac{2-\chi}{\chi} \sigma_{\tau n}+\frac{m_{\tau n n}}{2}+\frac{q_{\tau}}{5}\right), \\
\theta=\theta_{w}-\frac{1}{p_{\chi}}\left(\sqrt{\frac{\pi}{2} \theta} \frac{2-\chi}{\chi} \frac{q_{n}}{2}+\frac{\theta \sigma_{n n}}{4}+\frac{5 R_{n n}}{56}+\frac{\Delta}{30}\right)+\frac{\left(v_{\tau}-v_{w}\right)^{2}}{4}, \\
R_{\tau n}=\sqrt{\frac{2}{\pi \theta}} \frac{\chi}{2-\chi}\left[p_{\chi} \theta\left(v_{\tau}-v_{w}\right)-\frac{1}{2} \theta m_{\tau n n}-\frac{11}{5} \theta q_{\tau}-p_{\chi}\left(v_{\tau}-v_{w}\right)^{3}+6 p_{\chi}\left(\theta-\theta_{w}\right)\left(v_{\tau}-v_{w}\right)\right], \\
m_{\tau \tau n}=-\sqrt{\frac{2}{\pi \theta}} \frac{\chi}{2-\chi}\left[\frac{p_{\chi}}{5}\left(\theta-\theta_{w}\right)-\frac{4 p_{\chi}}{5}\left(v_{\tau}-v_{w}\right)^{2}+\frac{R_{\tau \tau}}{14}+\theta\left(\sigma_{\tau \tau}-\frac{\sigma_{n n}}{5}\right)+\frac{\Delta}{150}\right], \\
m_{n n n}=\sqrt{\frac{2}{\pi \theta}} \frac{\chi}{2-\chi}\left[\frac{2 p_{\chi}}{5}\left(\theta-\theta_{w}\right)-\frac{R_{n n}}{14}+\frac{\Delta}{75}-\frac{7}{5} \theta \sigma_{n n}-\frac{3 p_{\chi}}{5}\left(v_{\tau}-v_{w}\right)^{2}\right],
\end{gathered}
$$

where $\chi$ is accommodation coefficient for Maxwell accommodative model [28] and $p_{\chi}=p+\frac{\sigma_{n n}}{2}-\frac{R_{n n}}{28 \theta}-\frac{\Delta}{120 \theta}$.

\section{NUMERICAL METHODS}

The variant of high resolution Godunov scheme with linear flow parameter reconstruction is chosen for numerical solution of the R13 set of equations [21]. The second-order accuracy in space for smooth solutions is achieved using essentially two-dimensional reconstruction procedures $[12,21]$ for the primitive variables within each computational cell. The standard central difference approximation is used for elliptical part discretization of the fluxes (diffusion terms). Six kinetic boundary conditions are taken as a base of mathematical model of the gas-wall interaction. The complete system of the wall boundary conditions is obtained by the adding of the approximations of the selected subset of the R13 bulk equations [30,12] for the calculation of boundary convective flux. The resulted set of nonlinear equations for the wall is solved with Newtons numerical method [12].

Direct Simulation Monte Carlo (DSMC) computations are performed with SMILE++ software system [23, 24] that uses the majorant frequency scheme [31].

\section{RESULTS OF NUMERICAL SIMULATION}

The computational domain is presented in Figure 1. As it mentioned above, the Knudsen number based on the plate length is equal to $\mathrm{Kn}=0.05$. Figure 2 presents the comparison of the distributions of the local Mach number obtained by R13 (Fig. 2a is the linear variant, Eqs. (12), and Fig. 2b is the nonlinear one, Eqs. (8) - (10)). First of all, there is the strongest difference between the results of the linear and nonlinear versions of the R13 system with the DSMC data at the leading edge of the plate. This area is the most difficult in this problem. The greatest gradients of all macro-parameters of the flow are located right here. At the same time, this implementation of the boundary conditions for the R13 system has not yet been tested in detail for similar large values of local derivatives near the wall. Fig. 3 demonstrates an overpredicted increase in gas temperature in this region, which noticeably pushes the shock wave away from the plate. If we go further downstream from this area, we can see that the isolines of the Mach number, temperature, and other gas macroparameters are getting closer to the DSMC result. Moreover, an interesting fact is 


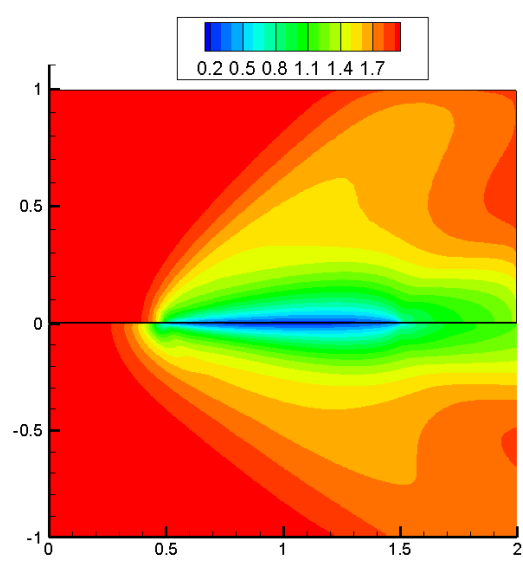

a

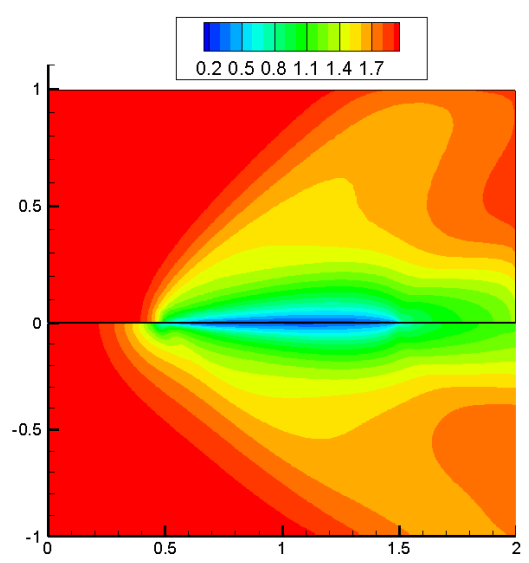

b

FIGURE 2. $M a_{\infty}=2.0$. Local Mach number distribution comparison of R13 (a - linear, $\mathrm{b}$ - nonlinear) results with DSMC data (upper parts of the Figures).

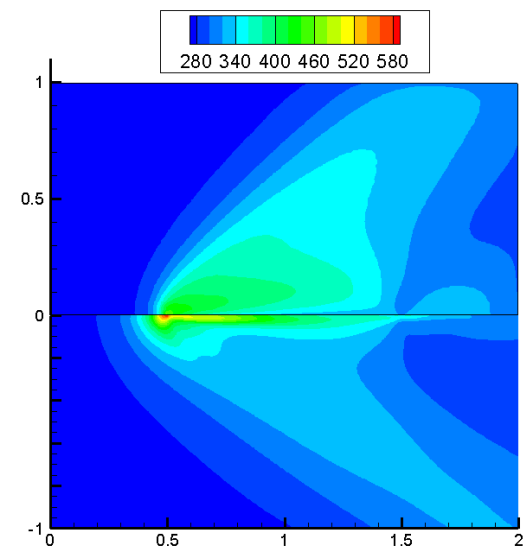

a

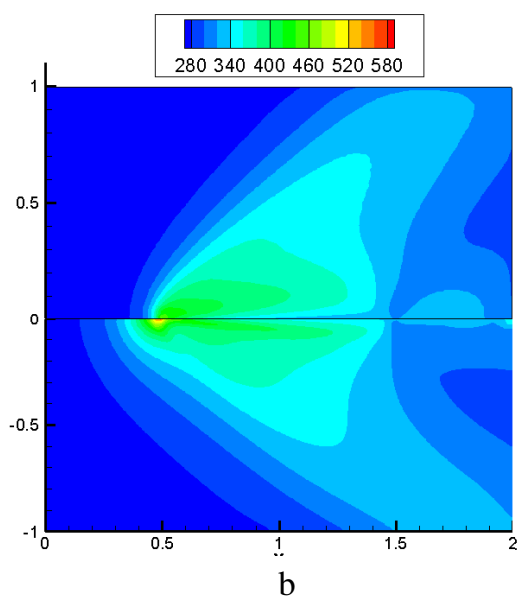

b

FIGURE 3. $M a_{\infty}=2.0$. Temperature distribution comparison of R13 ( $\mathrm{a}$ - linear, $\mathrm{b}$ - nonlinear) results with DSMC data (upper parts of the Figures).

that the linear version of R13 behaves noticeably worse than the nonlinear one even with the Mach number of the free-stream Mach number $M a=2.0$. This seems unusual due to the fact that in [9] it was shown that all variants of R13 allow to obtain almost identical distributions of macro-parameters in the one-dimensional internal structure of the shock wave with $M a \leq 2.0$.

There is the same situation in case of $M a_{\infty}=4.0$. The solution of linear R13 version is worse than R13 variant with non-gradient transport terms (NGMT). The zoomed region around the leading edge of plate is presented in Figures 4 and 5. As it can be seen, the difference between the solutions increases with the increase of free-stream Mach number. The shock wave offset from plate is also noticeably bigger. At the same time the nonlinear variant gives better qualitative results in the zone farthest from the front edge of the plate.

\section{CONCLUSION}

The problem of supersonic flow over the flat plate has been solved by our implementation R13 linear and nonlinear modifications. There are two two facts which arise here. The first one is the opened question of boundary conditions on a solid wall in case of large gradients of macro-parameters. It is the question for the future study. It is not clear now 


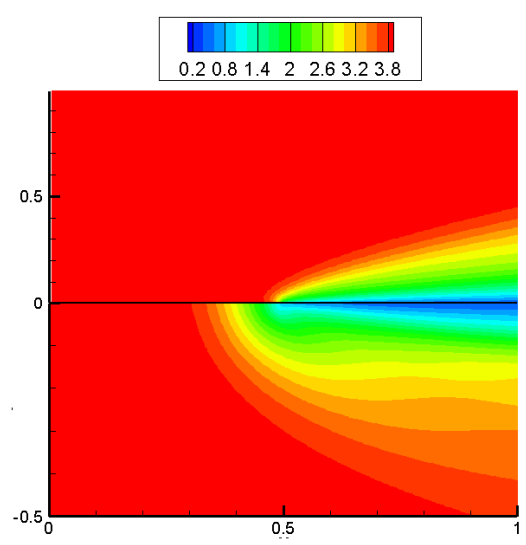

a

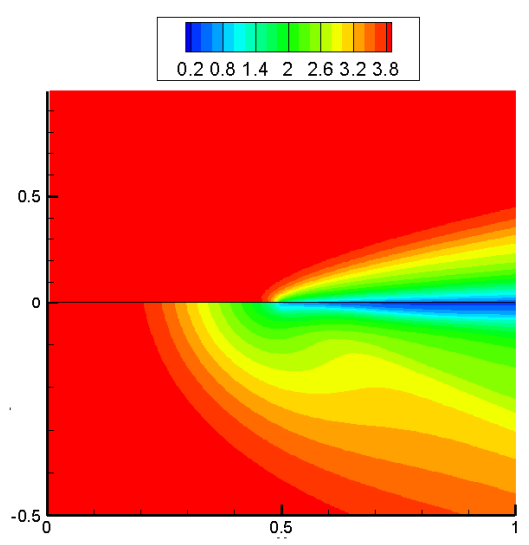

b

FIGURE 4. $M a_{\infty}=4.0$. Local Mach number distribution comparison of R13 (a - linear, $\mathrm{b}$ - nonlinear) results with DSMC data (upper parts of the Figures).

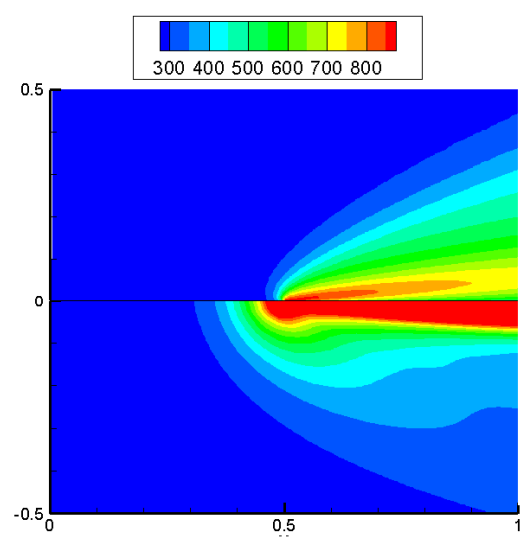

a

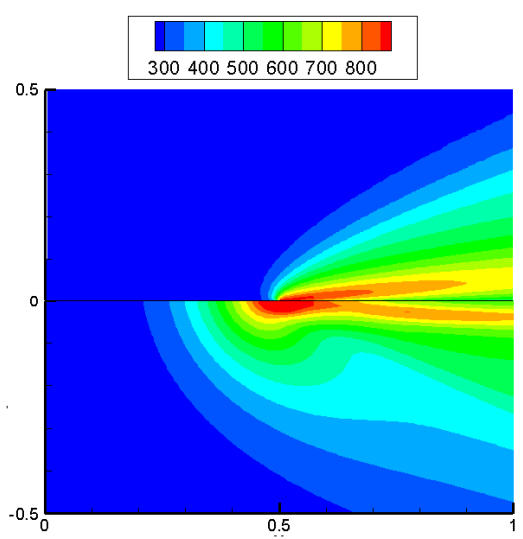

b

FIGURE 5. $M a_{\infty}=4.0$. The temperature distribution comparison of R13 ( $\mathrm{a}$ - linear, $\mathrm{b}$ - nonlinear) results with DSMC data (upper parts of the Figures).

if it is the numerical or mathematical problem here because almost all problems which were solved by R13 equations before (especially slow-velocity flows) had not such difficulties in near-boundary area for wall-boundary conditions $[10,11,12,13,15]$. The second conclusion here is the obvious difference of both R13 variants even in the case of $M a=2.0$ free-stream Mach number. It is led us to the idea that the nonlinear R13 variant becomes more preferable for supersonic calculations even for moderate Mach number flows.

\section{ACKNOWLEDGMENTS}

The work carried out at the Moscow State University was supported by the Russian Foundation for Basic Research (project No. 16-31-60034). The work carried out at the Institute of Theoretical and Applied Mechanics was supported by the Russian Science Foundation (Grant No. 17-19-01375). The work carried out at the University of Victoria was supported by and the Natural Sciences and Engineering Research Council (NSERC). 


\section{REFERENCES}

[1] H. Grad, Communications on Pure and Applied Mathematics 2, 331-407 (1949).

[2] L. H. Holway, Phys. Fluids 7, 911-213 (1964).

[3] M. Kogan, Rarefied gas dynamics (Plenum, New York, 1969).

[4] H. Grad, Pure Appl. Math 5, 257-300 (1952).

[5] M. Torrilhon and H. Struchtrup, Journal of Fluid Mechanics 513, p. 171198 (2004).

[6] H. Struchtrup and M. Torrilhon, Phys. Fluids 15, 2668-2680 (2003).

[7] X. J. Gu and D. R. Emerson, J. Fluid Mech. 636, 177-216 (2009).

[8] M. Y. Timokhin, H. Struchtrup, A. A. Kokhanchik, and Y. A. Bondar, AIP Conf. Proceedings 1786, p. 140006 (2016).

[9] M. Y. Timokhin, H. Struchtrup, A. A. Kokhanchik, and Y. A. Bondar, Phys. Fluids 29, p. 037105 (2017).

[10] H. Struchtrup and M. Torrilhon, J. Comput Phys. 227, 1982-2011 (2008).

[11] P. Taheri and H. Struchtrup, Phys. Rev. E 80, p. 066317 (2009).

[12] I. E. Ivanov, I. A. Kryukov, and M. Y. Timokhin, Comput. Math. Math. Phys. 53, 1534-1550 (2013).

[13] M. Y. Timokhin, I. E. Ivanov, and I. A. Kryukov, AIP Conf. Proceedings 1628, 748-755 (2014).

[14] A. S. Rana and H. Struchtrup, Phys. Fluids 28, p. 027105 (2016).

[15] M. Torrilhon, Annu. Rev. Fluid Mech. 48, 429-458 (2016).

[16] A. Beckmann, A. Rana, M. Torrilhon, and H. Struchtrup, Entropy 20, p. 680 (2018).

[17] J. Baliti, M. Hssikou, and M. Alaoui, Heat Transfer-Asian Res. 47, 1-20 (2018).

[18] H. Struchtrup, Macroscopic transport equations for rarefied gas flows (Springer, 2005).

[19] M. Torrilhon, Multiscale Model. Simul. 5, 695-728 (2006).

[20] I. A. Znamenskaya, I. E. Ivanov, I. A. Kryukov, I. V. Mursenkova, and M. Y. Timokhin, Tech. Phys. Lett. 40, 533-536 (2014).

[21] M. Y. Timokhin, Y. A. Bondar, A. A. Kokhanchik, M. S. Ivanov, I. E. Ivanov, and I. A. Kryukov, Phys. Fluids 27, p. 037101 (2015).

[22] M. Y. Timokhin, I. E. Ivanov, and I. A. Kryukov, Journal of Physics: Conference Series 1009, p. 012033 (2018).

[23] M. Ivanov, A. Kashkovsky, S. Gimelshein, G. Markelov, A. Alekseenko, Y. Bondar, G. Zhukova, S. Nikiforov, and P. Vashchenkov, Proceedings of 25th International symposium on rarefied gas dynamics. SaintPetersburg, Russia 539-544 (2006).

[24] A. V. Kashkovsky, Y. A. Bondar, G. A. Zhukova, M. S. Ivanov, and S. F. Gimelshein, AIP Conference Proceedings 762, 583-588 (2005).

[25] G. A. Bird, Molecular Gas Dynamics and The Direct Simulations of Gas Flows (Oxford Univesity Press, 1994).

[26] S. Chapman and T. G. Cowling, The mathematical theory of non-uniform gases: an account of the kinetic theory of viscosity, thermal conduction and diffusion in gases (Cambridge mathematical library, 1991).

[27] X. J. Gu and D. R. Emerson, J. Comput. Phys. 225, 263-283 (2007).

[28] J. C. Maxwell, Phil. Trans. R. Soc. (London) 170, 231-256 (1879).

[29] H. Grad, Principles of the kinetic theory of gases, volume 12 of Handbuch der Physik (Springer, 1958).

[30] M. Y. Timokhin, I. E. Ivanov, and I. A. Kryukov, AIP Conf. Proceedings 1501, 843-848 (2012).

[31] M. Ivanov and S. Rogasinsky, Soviet Journal of Numerical Analysis and Mathematical Modeling 3, 453-465 (1988).

[32] J. C. Maxwell, Phil. Trans. R. Soc. (London) 170, 231-256 (1879). 